\title{
FIXED POINTS OF PRODUCTS AND ORDERED SUMS OF SIMPLY ORDERED SETS
}

\section{SEYMOUR GINSBURG}

Let $A$ and $B$ be simply ordered sets and $A \times B$ the cartesian product ordered by first differences. Sufficiency conditions are given on the sets $A$ and $B$ for the existence of fixed points in the set $A \times B$ (Theorem 2). Sufficiency conditions are given on the sets $A, B$, and $C$ for the incomparability of the order types ${ }^{1}|A \times B|$ and $|A \times C|$ $(|C \times A|$ and $|C \times B|$ ) (Corollary 2 of Theorem 7 ). The content of Theorem 4 is that if $\left\{A_{\xi}\right\}$ is a family of $2^{\aleph_{0}}$ linear sets, each containing a fixed point, then there exists a subset $G$ of $R \times R$, where $R$ denotes the real numbers, such that for each element $x$ in $R$, if $B_{x}$ $=\{y \mid(x, y) \in G\}$, either $B_{x}$ is empty or is one of the sets $A_{\xi}$. Furthermore, if $p$ is a fixed point in $B_{x}$, then $(x, p)$ is a fixed point in $G$.

1. Fixed points of ordered sums and products. The element $p$ of the simply ordered set $A$ is said to be a fixed point of $A$ if $f(p)=p$ for every similarity transformation $f$ of $A$ into $A$.

Lemma 1. Let $p$ and $q$ be fixed points of the disjoint simply ordered sets $A$ and $B$ respectively. Then either $p$ or $q$ is a fixed point of the ordered sum, $C=A+B .^{2}$

Proof. Assume the contrary, i.e., assume that there exist similarity transformations $f$ and $g$ of $C$ into $C$ for which $f(p) \neq p$ and $g(q) \neq q$. Suppose that $f(p)<p$. Then the function $h$, which is defined by $h(x)$ $=x$ for $x>p, x$ in $A$, and $h(x)=f(x)$ for $x \leqq p$, is a similarity transformation of $A$ into $A$ for which $h(p) \neq p$. From this contradiction of $p$ being a fixed point in $A$, we see that $f(p)>p$. Analogous arguments show that $g(q)<q, f(q) \leqq q$, and $g(p) \geqq p$. Let $k$ be the similarity transformation of $C$ into $C$ which is defined by $k(x)=f(x)$ for $x<q$ and $k(x)=x$ for $x \geqq q$. This shows that without loss of generality we

Presented to the Society, September 5, 1952 under the title, $A$ theorem on similarity transformations, and November 29, 1952 under the title Some results on fixed points of simply ordered sets; received by the editors December 18, 1952 and, in revised form, October 23, 1953.

${ }^{1}$ If $A$ is a simply ordered set, $|A|$ denotes its order type.

${ }^{2}$ Let $B$ be a simply ordered set. If $\left\{A_{b} \mid b \in B\right\}$ is a family of pairwise disjoint simply ordered sets, then the ordered sum, $\sum_{b} \in_{B} A_{b}$, is the set union of the $A_{b}$, the elements being ordered as follows. If $c$ and $d$ are two distinct elements in $A_{b}$, then $c$ and $d$, considered in the ordered sum, have the same order as in the set $A_{b}$. If $c$ is in $A_{a}$ and $d$ is in $A_{b}$, where $a<b$, then $c<d$. 
may suppose that $f(x)=x$ for $x \geqq q$, and likewise, that $g(x)=x$ for $x \leqq p$.

Suppose that $f g(B)$ is a subset of $B$. As $q$ is a fixed point in $B$, $f g(q)=q$. On the other hand, since $g(q)<q$, it follows that $f g(q)<f(q)$ $=q$. From this contradiction we conclude that $f g(B) \cap A$ is nonempty, and thus that $f g(A)$ is a proper subset of $A$.

Since $f g$, acting on $A$, is a similarity transformation of $A$ into $A$, $f g(p)=p$. But $f[g(p)]=f(p)>p$. This is a contradiction. Thus either $p$ or $q$ is a fixed point in $C$. Q.E.D.

REMARK. It is not true, in general, that both $p$ and $q$ are fixed points in $C$. One need only consider the case where $A=\{\xi \mid \xi \leqq \omega\}, B$ $=\{\xi \mid \omega<\xi \leqq \omega+\omega\}, p=\omega$, and $q=\omega+\omega$.

Corollary. If $\left\{A_{1}, A_{2}, \cdots, A_{n}\right\}$ are a family of pairwise disjoint sets, and $p_{j}$ is a fixed point in $A_{j}$, then at least one of the points $p_{j}$ is a fixed point in the ordered sum, $C=A_{1}+\cdots+A_{n}$.

Theorem 1. Let $\left\{A_{1}, A_{2}, \cdots, A_{n}\right\}$ be a family of pairwise disjoint sets such that ${ }^{3}\left|A_{i}\right| \equiv\left|A_{j}\right|$ for $i, j \leqq n$. For $i=2,3, \cdots, n$, let $f_{i}$ be a similarity transformation of $A_{1}$ into $A_{i}$. Let $p$ be a fixed point of $A_{1}$, and $B$ the ordered sum, $A_{1}+A_{2}+\cdots+A_{n}$. (a) If $p$ is a fixed point in $A_{1} \cup A_{2}$, then $p$ is a fixed point in $B$, and thus a fixed point in $\cup_{i \leqq j} A_{i}$. If $f_{2}(p)$ is a fixed point in $A_{1} \cup A_{2}$, then $f_{n}(p)$ is a fixed point in $B$. (b) If both $p$ and $f_{2}(p)$ are fixed points in $A_{1} \cup A_{2}$, then each point $f_{i}(p)=p_{i}$ is a fixed point in $B$.

Proof. Using induction suppose that the theorem has been demonstrated for $n=2,3, \cdots, k$.

To prove the first sentence in (a) suppose that $p$ is not a fixed point in the set $B=\bigcup_{i \leqq k+1} A_{i}$, i.e., there is a similarity transformation $f$ of $B$ into $B$ for which $f(p) \neq p$. Let $m$ be the largest integer $\leqq k$ for which $f\left(p_{j}\right) \neq p_{j}$ for $j \leqq m$. If $m<k$, then $f\left(p_{m+1}\right)=p_{m+1}$. Thus the function $g$ on $C=\bigcup_{i \leqq k} A_{i}$, which is defined by $g(x)=f(x)$ for $x<p_{m+1}$ and $g(x)=x$ for $x \geqq p_{m+1}$, is a similarity transformation. Since $g(p) \neq p$, our induction hypothesis that $p$ is a fixed point in $C$ is contradicted. Now suppose that $m=k$. As is easily seen from our induction hypothesis, $f\left(p_{m}\right)>p_{m}$. Let $D=A_{k} \cup A_{k+1}$. Let $h$ be the similarity transformation of $D$ into $D$ which is defined by $g(x)=x$ for $x<p_{m}$, and $g(x)=f(x)$ for $x \geqq p_{m}$. Since $\left|A_{i}\right| \equiv\left|A_{j}\right|$, from I of $\S 2$ of $[4], p_{m}$ is a fixed point in $D$. But $g\left(p_{m}\right) \neq p_{m}$. From this contradiction we see that $p$ must be a fixed point in $B$.

${ }^{3}\left|A_{i}\right| \equiv\left|A_{j}\right|$ if there exists a similarity transformation of $A_{i}$ into $A_{j}$, and a similarity transformation of $A_{j}$ into $A_{i}$. 
To see the second sentence in (a) let $g_{2}$ be a similarity transformation of $A_{2}$ into $A_{1}$. Since $g_{2} f_{2}$ is a similarity transformation of $A_{1}$ into $A_{1}$ and $p$ is a fixed point in $A_{1}, g_{2}\left[f_{2}(p)\right]=p$. Let $\sigma$ be the similarity transformation of $A_{1} \cup A_{2}$ into $A_{k} \cup A_{k+1}$ which is defined by $\sigma(x)$ $=f_{k}(x)$ for $x$ in $A_{1}$ and $\sigma(x)=f_{k+1} g_{2}(x)$ for $x$ in $A_{2}$. Since $\left|A_{1} \cup A_{2}\right|$ $\equiv\left|A_{k} \cup A_{k+1}\right|$, by I of $\S 2$ of $[4], \sigma\left[f_{2}(p)\right]=f_{k+1}(p)$ is a fixed point in $A_{k} \cup A_{k+1}$. By a method analogous to that in the preceding paragraph one shows that $p_{k+1}$ is fixed in $A_{k-1} \cup A_{k} \cup A_{k+1}$, then fixed in $A_{k-2} \cup A_{k-1} \cup A_{k} \cup A_{k+1}$, etc, and finally, fixed in $\bigcup_{j \leqq k+1} A_{j}$.

To see part (b) suppose that both $p$ and $p_{2}$ are fixed points in the set $A_{1} \cup A_{2}$. Furthermore, suppose that for some $j$, there exists a similarity transformation $f$ of $B$ into $B$ for which $f\left(p_{j}\right) \neq p_{j}$. Clearly $j<n$. If $f\left(p_{j}\right)<p_{j}$, then it is easy to construct a similarity transformation $g$ of $\bigcup_{i \leqq j} A_{i}$ into $\bigcup_{i \leqq j} A_{i}$ for which $g\left(p_{j}\right) \neq p_{j}$. This contradicts part (a) for $n=j$. If $f\left(p_{j}\right)>p_{j}$, then there exists a similarity transformation $h$ of $\bigcup_{j \leqq i \leqq n} A_{i}$ into $\bigcup_{j \leqq i \leqq n} A_{i}$ for which $h\left(p_{j}\right) \neq p_{j}$. By proceeding as in the previous paragraph we see that $p_{j}$ is fixed in $A_{j} \cup A_{j+1}$. On applying (a) we see that $p_{j}$ is fixed in $\bigcup_{j \leqq i \leqq n} A_{i}$. Since $h\left(p_{j}\right) \neq p_{j}$ we have a contradiction. Therefore $f\left(p_{j}\right)=p_{j}$. Q.E.D.

We now consider the existence of fixed points in the set $A \times B$, where $A \times B$ is ordered by first differences, i.e., $(a, b)<(c, d)$ if $a<c$, or if $a=c$ and $b<d$. It is obvious that if either $A$ or $B$ contains no fixed points, then $A \times B$ contains none. The question arises as to what occurs in the case where $A$ and $B$ both contain fixed points.

THEOREM 2. Let $p$ and $q$ be fixed points respectively of the simply ordered sets $A$ and $B$. Then there exists a fixed point $(r, q)$ of $A \times B$, where $r$ is some fixed point of $A$ such that both of the sets

$$
\{x \mid r \leqq x \leqq p, x \in A\} \quad \text { and } \quad\{x \mid p \leqq x \leqq r, x \in A\}
$$

are finite.

Proof. For two points $a$ and $b$ in $A$ let $a R b$ if both of the sets

$$
\{x \mid a \leqq x \leqq b, x \in A\} \text { and }\{x \mid b \leqq x \leqq a, x \in A\}
$$

are finite. Let $N_{a}$ be the equivalence class, determined by the equivalence relation $R$, which contains the element $a$. The order type of the set $N_{a}$ is either $\omega, \omega^{*}, \omega^{*}+\omega$, or $n$, where $n$ is finite.

Now let $f$ be a similarity transformation of $A \times B$ into $A \times B$. Suppose that the order type of the set $N_{a}$ is $\omega$, i.e., the elements of $N_{a}$ are $y_{1}<y_{2}<\cdots<y_{n}<\cdots$. Let $E$ be the set of $A$ coordinates of the elements of the set $f\left(N_{a} \times B\right)$. Since $q$ is a fixed point of $B$, by Lemma 
1.3 of $[4]^{4},|m \times B|<|(m+1) \times B|$ for each positive integer $m$. Thus the set $E$ is infinite. Let $z_{1}<z_{2}<\cdots<z_{n}<\cdots$ be a sequence of points of $E$. Let $g\left(y_{n}\right)=z_{4 n+2}$ if $y_{n} \neq z_{4 n+2}$, and $g\left(y_{n}\right)=z_{4 n+3}$ if $y_{n}$ $=z_{4 n+2}$. If the order type of the set $N_{a}$ is $\omega^{*}$, or $\omega^{*}+\omega$, then $g$ can be defined analogously to the above, so that $g(x) \neq x$ for each $x$ in $N_{a}$. Finally, suppose that the order type of the set $N_{a}$ is $n$, where $n$ is finite. Again let $E$ be the set of $A$ coordinates of elements in the set $f\left(N_{a} \times B\right)$. Let $F=\{x \mid c<x<d, c \in E, d \in E, x \in A\}$ and $G=E \cup F$. Since $|B||j|<|B||(j+1)|$ the power of the set $G$ is at least $n$. If the power of the set $G$ is greater than $n$, then a similarity transformation $g$, of $N_{a}$ into $G$, can be defined in such a manner that $g(x) \neq x$. If the power of $G$ is exactly $n$, then a similarity transformation $g$, from $N_{a}$ to $G$, can be defined in precisely one way.

The function $g$ is thus defined to be one-to-one on each set $N_{a}$, and as is easily seen, is also one-to-one on all of $A$. Clearly $g$ preserves the order of the elements. Thus $g$ is a similarity transformation of $A$ into $A$. As $p$ is a fixed point of $A, g(p)=p$. This implies that $N_{p}$ is finite and the set $G$ that is associated with $N_{p}$ contains exactly as many elements as $N_{p}$. Since each element $y$ in $N_{p}$ is also a fixed point in $A$ (IV of $\S 2$ of [4]), $g(y)=y$. This shows that $f\left(N_{p} \times B\right)$ is a subset of $N_{p} \times B$. This is also true for any other similarity transformation $h$ of $A \times B$ into $A \times B$. Since $N_{p} \times B$ is the ordered sum of $n$ disjoint sets which are similar to $B, n$ being the number of elements in $N_{p}$, by the Corollary to Lemma 1 , one of the points $(r, q)$, where $r$ is some element in $N_{p}$, is a fixed point. Q.E.D.

If each point of $B$ is a fixed point of $B$, then the conclusion of Theorem 2 can be strengthened. First, however, we prove a general result on similarity transformations.

Lemma 2. Let $g$ be a similarity transformation of $C \times D$ into $E \times F$ which has the property that for each element $a$ in $C$, there are at least two elements $u(a)$ and $v(a), u(a)<v(a)$, in $E$ such that

$$
g(a \times D) \cap(u(a) \times F) \neq \varnothing \text { and } g(a \times D) \cap(v(a) \times F) \neq \varnothing .
$$

Then (a) for a given element $p$ in $C$ and a given element $q$ in $E$, where $u(p) \leqq q \leqq v(p)$, a similarity transformation $h$ of $C$ into $E$ can be found so that $h(p) \neq q$, and for each element $a$ in $C$, either $h(a)=u(a)$ or $h(a)$ $=v(a)$; and (b) if $C=E$, then $h$ can be chosen so that $h(a) \neq a$ and $u(a) \leqq h(a) \leqq v(a)$.

Proof. Let $[u(a), v(a)]=\{x \mid u(a) \leqq x \leqq v(a), x \in E\}$, and $W$

${ }_{4}|A|<|B|$ if there exists a similarity transformation of $A$ into $B$, but no similarity transformation of $B$ into $A$. See [7, p. 253]. 
$=\{[u(a), v(a)] \mid a \in C\}$. Since $g$ is a similarity transformation, it follows that no element of $E$ can be in more than two elements of $W$. Furthermore, if the element $x$ is in two elements of $W$, say $w_{1}$ and $w_{2}$, then $w_{1}=[a, v]$ and $w_{2}=[z, a]$, or $w_{1}=[z, a]$ and $w_{2}=[a, v]$. Clearly if $a<b$, then $u(a)<v(a) \leqq u(b)<v(b)$.

Suppose that the two elements, $p$ in $C$ and $q$ in $E, u(p) \leqq q \leqq v(p)$, are given. If $u(p) \neq q$, let $h(x)=u(x)$ for $x$ in $C$. If $u(p)=q$, let $h(x)$ $=v(x)$ for $x$ in $C$. The function $h$ satisfies the conclusions in (a).

Now suppose that $C=E$. For any two elements $a$ and $b$ in $E$, let $a R b$ and $N_{a}$ have the same meaning as in Theorem 2. Suppose that the order type of $N_{a}$ is $\omega$, the elements of $N_{a}$ being $y_{0}<y_{1}<\cdots$. It follows from the definition of the set $N_{a}$ and the fact that the $y_{n}$ are a sequence of consecutive elements in $E$ that $[u(y), v(y)] \cap\left[u\left(y_{n}\right)\right.$, $\left.v\left(y_{n}\right)\right]=\varnothing$ for any element $y$ in $E-N_{a}$. Let $h\left(y_{0}\right)=u\left(y_{0}\right)$ if $u\left(y_{0}\right) \neq y_{0}$, and $h\left(y_{0}\right)=v\left(y_{0}\right)$ if $u\left(y_{0}\right)=y_{0}$. Suppose that $h\left(y_{i}\right)$ has been defined for $i \leqq j$. Two possibilities occur. Either $v\left(y_{j}\right)<u\left(y_{j+1}\right)$ or $v\left(y_{j}\right)=u\left(y_{j+1}\right)$. Consider the first alternative. Let $h\left(y_{j+1}\right)=u\left(y_{j+1}\right)$ if $u\left(y_{j+1}\right) \neq y_{j+1}$, and $h\left(y_{j+1}\right)=v\left(y_{j+1}\right)$ if $u\left(y_{j+1}\right)=y_{j+1}$. Consider the second alternative. If $v\left(y_{j+1}\right) \neq y_{j+1}$, let $h\left(y_{j+1}\right)=v\left(y_{j+1}\right)$. Suppose that $v\left(y_{j+1}\right)=y_{j+1}$. As $y_{j}$ and $y_{j+1}$ are consecutive elements in $E, v\left(y_{j}\right) \leqq y_{j}$. If $v\left(y_{j}\right)=y_{j}$, then from our induction hypothesis that $h\left(y_{j}\right) \neq y_{j}$, and $h\left(y_{j}\right) \leqq v\left(y_{j}\right)$, we see that $h\left(y_{j}\right)<y_{j}$. In this case, let $h\left(y_{j+1}\right)=u\left(y_{j+1}\right)=y_{j}$. If $v\left(y_{j}\right)$ $<y_{j}$, let $h\left(y_{j+1}\right)=y_{j}$. Note that $u\left(y_{j+1}\right) \leqq h\left(y_{j+1}\right) \leqq v\left(y_{j+1}\right)$. In this way the function $h$ is defined on $N_{a}$. If the order type of $N_{a}$ is $\omega^{*}, \omega^{*}+\omega$, or $n$, then an analogous argument is applicable. The function $h$ is thus defined on each set $N_{a}$, and hence on all of $E$. Clearly it is a similarity transformation and satisfies the conclusions in (b). Q.E.D.

A simply ordered set $E$ is called exact if $E$ is nonempty and if each point of $E$ is a fixed point.

Theorem 3. Let $p$ be a fixed point of $E$ and let $F$ be an exact set. Then for each element $y$ in $F,(p, y)$ is a fixed point of $E \times F$.

Proof. Let $g$ be any similarity transformation of $E \times F$ into itself. Let $a$ be any element of $E$ for which there exists an element $b$ such that $g(a \times F) \subseteq(b \times F)$. For each element $y$ in $F$, let $m(y)=z$, where $g[(a, y)]=(b, z)$. The function $m(y)$ is a similarity transformation of $F$ into $F$. As $F$ is exact, $m(y)=y$ for each element $y$ in $F$, i.e., $g[(a, y)]$ $=(b, y)$. Therefore $g(a \times F)=(b \times F)$.

Suppose that $g(p \times F)$ is not the set $(p \times F)$. Let

$$
B=\left\{a \mid a \in E, g(a \times F)=\left(b_{a} \times F\right)\right\} \text { and } G=\left\{b_{a} \mid a \in B\right\} .
$$


Let $H=E-B$ and $M=E-G$. Since $g(a \times F)=\left(b_{a} \times F\right)$ for each element $a$ in $B$, it follows that $g(H \times F) \subseteq(M \times F)$. Furthermore, for each element $y$ in $H$, there are two elements, $u(y)$ and $v(y), u(y)$ $<v(y)$, in $M$ such that

$$
g(y \times F) \cap(u(y) \times F) \neq \varnothing \text { and } g(y \times F) \cap(v(y) \times F) \neq \varnothing .
$$

Two possibilities arise. Either $p$ is in $B$ or $p$ is in $H$. Suppose that the first alternative occurs. Then $g(p \times F)=\left(b_{p} \times F\right)$, where $p \neq b_{p}$. An application of Lemma 2 yields a similarity transformation $h$ of $H$ into $M$ such that $u(x) \leqq h(x) \leqq v(x)$, and $h(x) \neq x$. Let $f$ be the function which is defined by $f(x)=h(x)$ for each element $x$ in $H$, and $f(a)=b_{a}$ for each element $a$ in $B$. The function $f$ is a similarity transformation of $E$ into $E$ for which $f(p) \neq p$. This is a contradiction of the fact that $p$ is a fixed point of $E$. Suppose that the second possibility occurs, i.e., $p$ is in $H$. Applying Lemma 2 we obtain a function $h$ of $H$ into $M$ for which $h(p) \neq p$, and $u(x) \leqq h(x) \leqq v(x)$ for each element $x$ in $H$. Let $f(x)=h(x)$ for $x$ in $H$ and $f(a)=b_{a}$ for $a$ in $B$. Again $f(p) \neq p$. Thus both alternatives lead to a contradiction. Consequently we must have $g(p \times F)=(p \times F)$, i.e., $g[(p, y)]=(p, y)$ for each element $y$ in $F$. Therefore $(p, y)$ is a fixed point of $E \times F$.

\section{Corollary. If $E$ and $F$ are both exact, then $E \times F$ is exact.}

2. Imbedding sets. The set of real numbers, ordered in the natural manner, is denoted by $R$. A subset of $R$ is called a linear set. By $\theta$ is meant the smallest ordinal number whose power is $2^{\aleph_{0}}$.

Let $A_{0}$ be a given linear exact set of power $2^{\aleph_{0}}$. For each ordinal number $\xi$ where $1 \leqq \xi<\theta$ let $A_{\xi}$ be a linear set which is similar to $A_{0}$. Well order the elements of $A_{0}$ into the sequence $\left\{x_{\xi}\right\}_{\xi<\theta}$ and let $G=\bigcup_{\xi<\theta}\left(x_{\xi} \times A_{\xi}\right)$. Consider the set $G$ so obtained. $G$ is a subset of $R \times R$. The order type of $G$ is $A_{0} \times A_{0}$, and so is exact by the corollary to Theorem 3. Furthermore, for each element $x$ in $R$, if $B_{x}$ $=\{y \mid(x, y) \in G\}$, then either $B_{x}$ is empty or else is one of the sets $A_{\xi}$. This suggests the question of whether or not there exists an exact subset $G$ of $R \times R$, which has the property that the family of sets $\left\{B_{x} \mid x \in R\right\}$ contains all linear exact sets. No such set $G$ can exist, however, since there are $2^{2 \aleph_{0}}$ exact linear sets and only $2^{\aleph_{0}}$ sets $B_{x}$. Now if we only demand that the family of sets $\left\{B_{x}\right\}$ contain a preassigned family of $2^{\aleph_{0}}$ exact linear sets, then the set $G$ does exist. More precisely we have

Theorem 4. Let $\left\{A_{\xi} \mid \xi<\theta\right\}$ be a family of nonempty linear sets $A_{\xi}$ such that for each $\xi,\left|A_{\xi}\right|<|R|$. Then there exists a subset $G$ of $R \times R$ with the following properties: 
(a) For each element $x$ in $R$, the set $B_{x}=\{y \mid(x, y) \in G\}$ is either empty or else is one of the sets $A_{\xi}$.

(b) For each set $A_{\xi}$ there is only one element $x$ in $R$ such that $B_{x}=A_{\xi}$.

(c) The set $T=\left\{x \mid B_{x} \neq \varnothing\right\}$ is exact.

(d) If $p$ is a fixed point in $B_{x}$, then $(x, p)$ is a fixed point in $G$.

Proof. The demonstration is divided into two cases.

(a) Suppose that only a denumerable number of the sets, say $\left\{A_{n} \mid n<\gamma \leqq \omega\right\}$, are of power $\geqq 2$ each. By $(a, b)$ is meant the open interval $\{x \mid a<x<b\}$ of $R$. Let $S$ denote the set of positive real numbers. For each $n<\gamma$ let $C_{n}$ be a subset of $(2 n, 2 n+1)$ which is similar to $A_{n}$. Let $f$ be a similarity transformation of $S$ into $S$ which is not the identity function on $(2 n+1,2 n+2)$ for some $n<\gamma$. Let $F$ be the family of all such functions $f$. The power of $F$ is $2 \aleph_{0}$. Denote by $f^{*}$ the inverse function of $f$. Well order the elements of $S$ and $F$ into the two sequences, $\left\{y_{\xi}\right\}_{\xi<\theta}$ and $\left\{f_{\xi}\right\}_{\xi<\theta}$, respectively. For each $\xi$ let

$$
D_{\xi}^{n}=\left\{x \mid f_{\xi}(x) \neq x, x \in(2 n+1,2 n+2)\right\}
$$

and $D_{\xi}=\cup_{n<\gamma} D_{\xi}^{n}$. Clearly each set $D_{\xi}$ contains some open interval of $S$, call it $J_{\xi}$. Since $\left|A_{n}\right|=\left|C_{n}\right|<|R|$, by Theorem 8 of [7], $\sum_{n<\gamma}\left|C_{n}\right|$ $<|R|$. Let $Z=\cup_{n<\gamma} C_{n}, p_{0}$ be the first element in the set $J_{0}-f_{0}^{*}(Z)$, and $q_{0}=f_{0}\left(p_{0}\right)$. The element $p_{0}$ certainly exists since, by Lemmas 3 and 4 of [7], if $|A|<|R|$ and $|B| \equiv|R|$, then the power of the set $B-A$ is $2^{\aleph_{0}}$. Now suppose that $\left\{p_{\xi}\right\}_{\xi<\mu}$ and $\left\{q_{\xi}\right\}_{\xi<\mu}$ where $\mu<\theta$ have been defined. If $G_{\mu}=Z \cup E_{\mu}$, where

$$
E_{\mu}=\left\{p_{\xi} \mid \xi<\mu\right\} \cup\left\{q_{\xi} \mid \xi<\mu\right\},
$$

then by Lemma 3 of [7], $\left|f_{\mu}^{*}\left(G_{\mu}\right) \cup E_{\mu}\right|<\bar{R}$. Let $p_{\mu}$ be the first element in the set $J_{\mu}-\left[f_{\mu}^{*}\left(G_{\mu}\right) \cup E_{\mu}\right]$ and $q_{\mu}=f_{\mu}\left(p_{\mu}\right)$. Let $Y=Z \cup\left\{p_{\xi} \mid \xi<\theta\right\}$.

Suppose that $p$ is either a fixed point in $C_{i}$ or $p$ is a point in $Y-Z$, which is not a fixed point in $Y$. Then there exists a similarity transformation $g$ of $Y$ into $Y$ such that $g(p) \neq p$. Either $p$ is in some set $C_{i}$, where $i<\gamma$, or else $p$ is in some set $(2 n+1,2 n+2)$, where $n<\gamma$. Assume that the former is the situation. Since $p$ is a fixed point of $C_{i}$, $g\left(C_{i}\right)$ is not a subset of $C_{i}$. This implies that there exists an element $q$ in $Y$ such that $g(q) \neq q$ and $q$ is in either $(2 i-1,2 i)$ or $(2 i+1,2 i+2)$. From this we see that $g$ is not the identity function on $Y$ $\cap\left(\cup_{n<\gamma}(2 n+1,2 n+2)\right)$. By a familiar argument, such as given in Theorem 3 of [2] and Theorem 2.4 in [4], it can be shown that each set $Y \cap(2 n+1,2 n+2)$, for $n<\gamma$, is a dense exact subset of $(2 n+1$, $2 n+2)$. From this it follows that the function $g$ can be extended to become an element $f_{v}$ of $F$. Therefore the element $g\left(p_{v}\right)=q_{v}$ is not in 
$Y$, so that $g$ does not map $Y$ into $Y$. From this contradiction we conclude that the point $p$ is fixed in $Y$.

A similar argument shows that if $W=\left\{p_{\xi} \mid \xi<\theta\right\} \cup\left\{w_{n} \mid n<\gamma\right\}$, where $w_{n}$ is some definite point in $C_{n}$, then each point of $W$ is fixed, i.e., $W$ is exact.

Now the power of the set $Y-Z$ is $2^{N_{0}}$. Well order the elements of the set $Y-Z$ into the sequence $\left\{x_{\xi}\right\}_{\gamma \leqq \xi<\theta}$. Denote by $G$ the set

$$
G=\bigcup_{n<\gamma}\left[\left(2 n+\frac{1}{2}\right) \times A_{n}\right] \cap\left\{\left(x_{\xi} \times A_{\xi}\right) \mid \gamma \leqq \xi<\theta\right\} .
$$

Evidently $|G|=|Y|$ since each set $A_{\xi}, \xi \geqq \gamma$, consists of one point. It is easy to verify that $G$ satisfies the conclusions of the theorem.

(b) Suppose that a nondenumerable number of the sets $A_{\xi}$ are of power $\geqq 2$ each. Let $C$ be a dense exact subset of $R$, of power $2^{N_{0}}$, which has the property that no two disjoint subsets of $C$, of power $2 \aleph_{0}$ each, are similar. Furthermore, let each point of $C$ be a $c$-condensation point of $C$, where by $x$ being a $c$-condensation point of $C$ is meant that each open interval of $R,(a, x)$ and $(x, b)$, meets the set $C$ in $2^{\aleph_{0}}$ points. The existence of such a set $C$ is guaranteed by Lemma 1 and Theorems 4 and 5 of [3]. Divide the family of sets $\left\{A_{\xi} \mid \xi<\theta\right\}$ into two disjoint families, $\left\{D_{\xi} \mid \xi<\mu \leqq \theta\right\}$ and $\left\{C_{\xi} \mid \xi<\theta\right\}$, where the first family is a nondenumerable number of sets, of power $\geqq 2$ each. As is well known the elements of $C$ can be divided into two disjoint sets, $M=\left\{u_{\xi} \mid \xi<\mu\right\}$ and $N=\left\{v_{v} \mid \nu<\theta\right\}$, such that $M$ is dense in $C$, and each element $u_{\xi}$ is a condensation point of $C$ (in the usual sense). Now denote by $G$ the set

$$
G=\bigcup_{\xi<\mu}\left(u_{\xi} \times D_{\xi}\right) \cup \bigcup_{v<\theta}\left(v_{v} \times C_{v}\right) .
$$

The only property of $G$ that is not self-evident is (d). To see that (d) is satisfied suppose the contrary, i.e., suppose that there exists a similarity transformation of $G$ into $G$ such that $f(p) \neq p$ for some element $p$ in $G$, where $p=(d, t)$ and $t$ is a fixed point in $B_{d}$. Since $t$ is a fixed point in $B_{d}$, it follows that for some element $(d, q)$ in the set $\left(d, B_{d}\right)$, the element of $f[(d, q)]$ is not in $\left(d \times B_{d}\right)$, i.e., $f[(d, q)]=(z, r)$ where $d \neq z$. Suppose that $z>d$. Denote by $P$ the set $P=\{x \mid d<x<z$, $x \in C\}$. Note that $f[(x, y)]>(x, y)$ for $(x, y)$ in $G$, where $x$ is in $P$. Since each element of $C$ is a $c$-condensation point of $C$, the power of $P$ is $2 s_{0}$. Suppose that for two elements $x_{1}$ and $x_{2}, x_{1}<x_{2}$, in $P$, there exists an element $x_{3}$ in $C$ such that

$$
f\left(x_{1} \times B_{x_{1}}\right) \cap\left(x_{3} \times B_{x_{3}}\right) \neq \varnothing \text { and } f\left(x_{2} \times B_{x_{2}}\right) \cap\left(x_{3} \times B_{x_{3}}\right) \neq \varnothing \text {. }
$$

This implies that for each element $u_{\xi}$ in $M \cap P, f\left(u_{\xi} \times D_{\xi}\right) \subseteq\left(x_{3} \times B_{x_{3}}\right)$. 
Now there are a nondenumerable number of such elements $u_{\xi}$, from the selection of the $u_{\xi}$ and $v_{\xi}$. Since each set $D_{\xi}$ contains at least two elements, it follows that the set $\left(x_{3} \times B_{x_{3}}\right)$ contains a nondenumerable number of disjoint intervals. But this is impossible since $\left(x_{3} \times B_{x_{3}}\right)$ is similar to a subset of $R$. Consequently, for any two elements $x_{1}$ and $x_{2}$ in $P, f\left(x_{1} \times B_{x_{1}}\right)$ and $f\left(x_{2} \times B_{x_{2}}\right)$ cannot meet the same set $\left(x_{3} \times B_{x_{3}}\right)$. This fact implies the following:

For each subset $\left(x \times B_{x}\right)$ of $G$, where $x$ is in $P$, select a definite element $w_{x}$ in $B_{x}$. Let $g$ be the function which is defined by $g(x)=y$, where $f\left[\left(x, w_{x}\right)\right]=(y, t)$. Then $g$ is a similarity transformation of $P$ in to $C-P$.

From this we obtain two similar, disjoint subsets of $C$, of power $2^{\aleph_{0}}$ each, $P$ and $g(P)$. This, in turn, contradicts the fact that $C$ does not contain two such sets. Therefore $f[(d, q)]>(d, q)$ is false. An analogous argument shows that $f[(d, q)]<(d, q)$ is also false. Hence we are forced to conclude that no such function $f$ can exist, i.e., the original point $p$ is fixed. Q.E.D.

COROLlary 1. Let $\left\{A_{\xi}\right\}_{\xi<\theta}$ be a sequence of nonempty linear sets such that $\left|A_{\xi}\right|<|R|$ for each $\xi$. Then there exists a subset $G$ of $R \times R$ which satisfies (a), (c), and (d) of Theorem 4, and for which there exists a oneto-one correspondence $h$ between the sets $\{\xi \mid \xi<\theta\}$ and the set $\left\{x \mid B_{x} \neq \varnothing\right\}$ such that $A_{\xi}=B_{h(\xi)}$.

COROLlary 2. Let $\left\{A_{\xi} \mid \xi<\mu \leqq \theta\right\}$ be a family of nonempty linear sets such that $\left|A_{\xi}\right|<|R|$ for each $\xi$. Then there exists a subset $G$ of $R \times R$ which satisfies (a), (c), and (d) of Theorem 4.

If each set $A_{\xi}$ contains at least one fixed point, then $\left|A_{\xi}\right|<|R|$. If each set $A_{\xi}$ is exact we obtain

Corollary 3. Let $\left\{A_{\xi} \mid \xi<\theta\right\}$ be a family of linear exact sets. Then there exists an exact subset $G$ of $R \times R$ satisfying (a), (b), and (c) of Theorem 4.

The assumption in Theorem 4 that $\left|A_{\xi}\right|<|R|$ for each $\xi$ cannot be removed. For example, let $A_{0}=R$ and $A_{\xi}=\left\{p_{\xi}\right\}, \xi \geqq 1$, where $p_{\xi} \neq p_{v}$ for $\xi \neq v$. Any subset $G$ of $R \times R$ satisfying (a) and (b) is of order type $\equiv|R|$, thus contains no fixed point, and so violates (d).

The conclusion of Corollary 2 cannot be strengthened to include (b). This is seen by the following example. Let $A_{0}$ be a linear set containing a fixed point. For each positive integer $i$ let $A_{i}$ be a linear set, similar to $A_{0}$, such that $A_{m} \neq A_{n}$ for $m \neq n$. Now suppose that a subset $G$ of $R \times R$ exists which satisfies (a) and (b). Then the set $T$ is denumerably infinite. But in Theorem 1 of [2] Dushnik and Miller have 
demonstrated that there is no denumerably infinite exact set. Thus (c) and (d) are not satisfied.

3. Related results. In this section some applications of Lemma 2 are given.

A simply ordered set $E$ is said to have the fixed point property if, for each similarity transformation $f$ of $E$ into itself, there exists an element $p$ of $E$ such that $f(p)=p$.

The ordered couple of simply ordered sets $(E, F)$ is called an $A$-pair if, for each pair of simply ordered sets $G$ and $H$, and similarity transformation $f$ of $E \times G$ into $F \times H$, there exist two elements, $p$ in $E$ and $q$ in $F$, such that $f(p \times G)$ is a subset of $(q \times H)$ [1].

From Lemma 2 there immediately follows

Theorem 5. If $A$ and $B$ are any two sets for which there is no similarity transformation of $A$ into $B$, then $(A, B)$ is an $A$-pair.

Turning to sets $E$ for which $(E, E)$ is an $A$-pair we have

Theorem 6. If E has the fixed point property, then $(E, E)$ is an $A$ pair.

Proof. Let $g$ be a similarity transformation of $E \times D$ into $E \times F$, and suppose that there are no two elements $a$ and $b$ in $E$ for which $g(a \times D)$ is a subset of $(b \times F)$. This means that for each element $a$ in $E$ there are two elements, $u(a)$ and $v(a), u(a)<v(a)$, such that

$$
g(a \times D) \cap(u(a) \times F) \neq \varnothing \text { and } g(a \times D) \cap(v(a) \times F) \neq \varnothing \text {. }
$$

Applying Lemma 2 we obtain a similarity transformation $h$ of $E$ into $E$ such that for each element $a$ in $E, h(a) \neq a$. This contradicts our assumption that $E$ has the fixed point property. It therefore follows that there must exist two elements $a$ and $b$ in $E$ such that $g(a \times D)$ is a subset of $(b \times F)$, i.e., $(E, E)$ is an $A$-pair.

Corollary 1. If E has a fixed point, then $(E, E)$ is an $A$-pair.

Let $E$ be a well ordered set of order type $\alpha$. Arens has shown that if $\alpha=\omega$, then $(E, E)$ is not an $A$-pair [1]. We now extend that result. We may suppose that $E$ is the set of ordinal numbers which are smaller than $\alpha$. Suppose that $\alpha$ is a limit number. Consider the mapping $f$ of $E \times\{0,1\}$ onto $E \times\{0\}$ which is defined as follows. For $\xi<\alpha$ and $\xi=\sigma_{\xi}+n_{\xi}$, where $\sigma_{\xi}=0$ or is a limit number, and $n_{\xi}$ is a finite integer, let $f[(\xi, 0)]=\left(\sigma_{\xi}+2 n_{\xi}, 0\right)$ and $f[(\xi, 1)]=\left(\sigma_{\xi}+2 n_{\xi}+1,0\right)$. As $\alpha$ is a limit number $f$ is a similarity transformation of $E \times\{0,1\}$ onto $E \times\{0\}$. As $f$ is one-to-one, there are no two elements $a$ and $b$ in $E$ for which $f(a \times\{0,1\})$ is a subset of $(b, 0)$. Thus $(E, E)$ is not 
an $A$-pair. Now suppose that $\alpha$ is a nonlimit number. Then the hypotheses of Corollary 1 of Theorem 6 are satisfied, so that $(E, E)$ is an $A$-pair. Summarizing the preceding discussion we get

Corollary 2. Let $E$ be a well ordered set of order type $\alpha$. Then $(E, E)$ is an A-pair if and only if $\alpha$ is not a limit number.

$|A|$ and $|B|$ are said to be incomparable if there is no similarity transformation of $A$ into $B$ and no similarity transformation of $B$ into $A$. It is easy to find examples in which $|A|$ and $|B|$ are incomparable, while for some nonempty set $C,|A \times C|$ and $|B \times C|(|C \times A|$ and $|C \times B|)$ are not. This is so in the case where $|A|=\omega,|B|=\omega^{*}$, and $|C|=\eta, \eta$ being the order type of the rational numbers ordered in the natural manner. In fact, for each pair of nonempty sets $A$ and $B$, there always exists a set $C$ such that $|A \times C| \equiv|B \times C|(|C \times A|$ $\equiv|C \times B|)$. For let $|D|=|A|+|B|$ and $|C|=|D| \eta$ [5]. Then $|C||C|=|C|$ and $|C| \equiv|A \times C| \equiv|B \times C|(|C| \equiv|C \times A| \equiv|C \times B|)$.

Theorem 7. Let $A$ and $B$ be any two sets for which there is no similarity transformation of $A$ into $B$. Then

(a) for any set $C$ which has the fixed point property, there is no similarity transformation of $C \times A$ into $C \times B$;

(b) for any set $C$ such that $|C|<|C| 2$, there is no similarity transformation of $A \times C$ into $B \times C$.

Proof. (a) Suppose that $f$ is a similarity transformation of $C \times A$ into $C \times B$. For each element $c$ in $C, f(c \times A)$ is not a subset of $(d \times B)$, for some element $d$ in $B$. This is so since there is no similarity transformation of $A$ into $B$. Thus there are at least two elements in $B, u(c)$ and $v(c), u(c)<v(c)$, such that

$$
f(c \times A) \cap(u(c) \times B) \neq \varnothing \text { and } f(c \times A) \cap(u(c) \times B) \neq \varnothing .
$$

By Lemma 2, a similarity transformation $h$ of $C$ into $C$ can be found so that $h(y) \neq y$ for each element $y$ in $C$. This contradicts the fact that the set $C$ has the fixed point property. Therefore no such similarity transformation $f$ can exist.

(b) Since $|C|<|C| 2$, it follows from Lemma 1.3 of [4] that $|C| m<|C|(m+1)$ for each positive integer $m$. Using this fact, it follows from an argument analogous to that given in Theorem 2 that if a similarity transformation $f$ of $A \times C$ into $B \times C$ existed, then a similarity transformation $h$ of $A$ into $B$ could be constructed. By hypothesis though, no such function $h$ exists. Hence our result. Q.E.D.

Corollary 1. If $|A|<|B|$ and $C$ has the fixed point property, then 
$|C \times A|<|C \times B|$. If $|A|<|B|$ and $|C|<|C| 2$, then $|A \times C|$ $<|B \times C|$.

CoRollary 2. If $|A|$ and $|B|$ are incomparable order types and $C$ has the fixed point property, then $C \times A$ and $C \times B$ are incomparable order types. If $A$ and $B$ are incomparable order types and $|C|<|C| 2$, then $|A \times C|$ and $|B \times C|$ are incomparable order types.

Corollary 3. If $|C|<|C| 2$ and $|A \times C| \equiv|B \times C|$, then $|A|$ $\equiv|B|$.

Naturally the question arises as to whether or not the conclusion of Theorem 7 remains valid if the hypotheses in (a) and (b) are reversed. The answer is in the negative as is now shown. For (a) let $|A|=2$, $|B|=1$, and $|C|=\omega$. Consider (b). A simply-ordered set $E$ is said to be complete if every subset $D$ of $E$ has both a greatest lower bound and a least upper bound, in $E$. It is known that a complete set has the fixed point property [6]. Therefore the set $C$, where $|C|=1+\lambda$ +1 , has the fixed point property. Let $|A|=\omega$ and $|B|=\omega^{*}$. Clearly there is no similarity transformation of $A$ into $B$. Yet $|A \times C|$ $=(1+\lambda+1) \omega \equiv \lambda \equiv(1+\lambda+1) \omega^{*}=|B \times C|$.

Using the methods of this paper one can show

ThEOREM 8. If $A$ and $B$ each have the fixed point property, then the ordered sum $A+B$ and the product $A \times B$ each have the fixed point property.

\section{BIBLIOGRAPHY}

1. R. Arens, Ordered sequence spaces, Portugaliae Mathematica vol. 10 (1951) pp. $25-28$.

2. B. Dushnik and E. W. Miller, Concerning similarity transformations of linearly ordered sets, Bull. Amer. Math. Soc. vol. 46 (1940) pp. 322-326.

3. S. Ginsburg, Order types and similarity transformations, submitted to Fund. Math.

4. - Some remarks on order types and decompositions of sets, Trans. Amer. Math. Soc. vol. 74 (1953) p. 514-535.

5. F. Hausdorff, Grundzüge der Mengenlehre, New York, 1949, p. $147 \mathrm{ff}$. 133.

6. B. Knaster, Annales de la Société Polonaise de Mathématique vol. 6 (1927) p.

7. W. Sierpinski, Types d'ordre des ensembles linéaires, Fund. Math. vol. 37 (1950) pp. 253-264.

UNIVERSITY OF MiAMI 